\title{
Using Drell-Yan forward-backward asymmetry to reduce PDF uncertainties in the measurement of electroweak parameters
}

\author{
A. Bodek ${ }^{\text {a }}$ J. Han, A. Khukhunaishvili, W. Sakumoto \\ Department of Physics and Astronomy, University of Rochester, Rochester, NY 14627-0171, USA
}

Received: 10 July 2015 / Accepted: 19 February 2016 / Published online: 2 March 2016

(C) The Author(s) 2016. This article is published with open access at Springerlink.com

\begin{abstract}
The uncertainties in parton distribution functions (PDFs) are the dominant source of the systematic uncertainty in precision measurements of electroweak parameters at hadron colliders (e.g. $\sin ^{2} \theta_{\text {eff }}\left(M_{Z}\right), \sin ^{2} \theta_{W}=1-M_{W}^{2} / M_{Z}^{2}$ and the mass of the $\mathrm{W}$ boson). We show that measurements of the forward-backward charge asymmetry $\left(A_{F B}(M, y)\right)$ of Drell-Yan dilepton events produced at hadron colliders provide a new powerful tool to reduce the PDF uncertainties in these measurements.
\end{abstract}

\section{Introduction}

Precision measurements in hadron colliders are limited by our knowledge of parton distribution functions (PDFs). In general, PDF fits by various groups including CTEQ [1,2], MMHT [3,4], NNPDF [5-7], HERA [8], and ABM [9] are extracted from fixed target experiments and various cross sections measurements at colliders. The fixed target experiments include electron, muon, neutrino, and Drell-Yan experiments. The collider experiments include ep(HERA), $\bar{p} p$ (Tevatron) and $p p$ (LHC).

Some of the fixed target measurements are on nuclear targets resulting in additional uncertainties from modeling of nuclear effects. Some of the fixed target measurements are also at low momentum transfers where the contributions of non-perturbative and higher twist effects may be significant. These issues are absent in collider cross section data. Therefore, recent PDF fits have placed a greater emphasis on collider cross section data.

1.1 Measurements of electroweak parameters at hadron colliders

Within the standard model, measurements of the mass of the $Z$ boson and top quark, in combination with the mass of the

a e-mail: bodek@pas.rochester.edu
Higgs boson, can be used to predict the mass of the $\mathrm{W}$ boson $\left(M_{W}\right)$. At present, the average of the all direct measurements of $M_{W}(80385 \pm 15 \mathrm{MeV})$ is about 1.5 standard deviation higher [10] than the prediction of the standard model. Predictions of supersymmetric models for $M_{W}$ are also higher than the predictions of the standard model [11]. Therefore, more precise measurements of the mass of $M_{W}$ are of great interest.

Alternatively, $M_{W}$ can also be extracted indirectly from measurements of the on-shell electroweak mixing angle $\sin ^{2} \theta_{W}$ by the relation $\sin ^{2} \theta_{W}=1-M_{W}^{2} / M_{Z}^{2}$.

Measurements of the forward-backward charge asymmetry in Drell-Yan dilepton events produced at hadron colliders (in the region of the $Z$ pole) have been used to measure the value of the effective electroweak (EW) mixing angle $\sin ^{2} \theta_{\text {eff }}^{\text {lept }}\left(M_{Z}\right)$ [12-15]. In addition, by incorporating electroweak radiative corrections in the analysis the CDF collaboration has also measured the on-shell EW mixing angle $\sin ^{2} \theta_{W}[12,13]$.

An uncertainty of \pm 0.00030 in the measurement of $\sin ^{2} \theta_{W}$ is equivalent to an indirect measurement of $M_{W}$ to a precision of $\pm 15 \mathrm{MeV}$. However, the PDF uncertainty quoted in the most recent measurement of $\sin ^{2} \theta_{\text {eff }}$ by the ATLAS collaboration [15] at the LHC is \pm 0.00090 . Therefore, a significant reduction in the PDF uncertainty is needed. In this communication, we show how $A_{F B}$ data also provide a new powerful tool to reduce PDF uncertainties in the measurements of electroweak parameters in hadron colliders

The constraints provided by $A_{F B}$ measurements in combination with constraints from the $\mathrm{W}$ charge asymmetry $\left(A_{W}\right)$ can be used to reduce the PDF uncertainty in the extracted value of $\sin ^{2} \theta_{W}$ and $\sin ^{2} \theta_{\text {eff }}^{\text {lept }}\left(M_{Z}\right)$ from $A_{F B}$ data. The $A_{F B}$ constraints on PDFs can also be used to reduce the PDF uncertainty in other precision measurements with $Z$ and $W$ bosons such as the measurement of $W_{W}$.

Asymmetries such as $A_{F B}$ and $A_{W}$ are ideal in providing additional constraints because asymmetries are less sensitive 
to the choice of QCD scale and QCD higher order terms. In addition, there are new techniques that can be used $[16,17]$ to greatly reduce the experimental systematic uncertainty in asymmetry measurements.

\section{$2 q \bar{q}$ annihilations to dileptons}

In leading order (LO) dileptons are primarily produced in quark-antiquark annihilation. Here, one parton (quark or antiquark) carries momentum $x_{1}$ and another parton carries momentum $x_{2}$. The momentum fractions $x_{1,2}$ carried by the partons are related to the mass (M) and rapidity (y) of the two leptons as follows:

$x_{1,2}=\frac{M}{\sqrt{s}} e^{ \pm y}$

The angular dependence of the differential cross section for $q \bar{q}$ annihilation to a dilepton pair can be written as

$\frac{d \sigma(M)}{d(\cos \theta)} \propto\left(1+\cos ^{2} \theta\right)+A_{4}(M) \cos \theta$

where $\theta$ is the emission angle of the negatively charged lepton relative to the quark momentum in the dilepton center of mass frame, and $A_{4}(M)$ is parameter that depend on the weak isospin and charge of the incoming quarks.

The cross sections for forward $\left(\sigma_{F}\right)$ and backward $\left(\sigma_{B}\right)$ events are given by

$$
\begin{aligned}
\sigma_{F}(M) & =\int_{0}^{1} \frac{d \sigma}{d(\cos \theta)} d(\cos \theta) \\
& \propto\left(1+\frac{1}{3}\right)+A_{4}(M)\left(\frac{1}{2}\right) \\
\sigma_{B}(M) & =\int_{-1}^{0} \frac{d \sigma}{d(\cos \theta)} d(\cos \theta) \\
& \propto\left(1+\frac{1}{3}\right)-A_{4}(M)\left(\frac{1}{2}\right)
\end{aligned}
$$

The electroweak interaction introduces an asymmetry (a linear dependence on $\cos \theta$ ), which can be expressed as

$$
A_{F B}(M)=\frac{\sigma_{F}-\sigma_{B}}{\sigma_{F}+\sigma_{B}}=\frac{3}{8} A_{4}(M)
$$

The dependence of $A_{F B}(M, y)$ on $\sin ^{2} \theta_{\text {eff }}^{\text {lept }}$ has been used to measure $\sin ^{2} \theta_{\text {eff }}^{\text {lept }}$ at the Tevatron and LHC.

The systematic uncertainties in the measurement of $A_{F B}(M, y)$ can be greatly reduced if $A_{F B}(M, y)$ is extracted from a measurement of $A_{4}(M, y)$. This can done by an event weighting technique [17] for which there is a cancelation of systematic errors that originate from uncertainties in acceptance and efficiencies. With this technique, no acceptance or efficiency corrections are needed. The extracted values of $A_{4}(M, y)$ using the event weighting technique are equal to the Born level $A_{4}(M, y)$. This technique has been using in the most recent measurements at $\mathrm{CDF}$ [13].

\section{$3 A_{F B}$ at the Tevatron}

For $\bar{p} p$ collisions, the direction of the quark is predominately in the proton direction, and the direction of the antiquark is predominately in the antiproton direction. Here, most of the cross section originates from the annihilation of quarks in the proton with antiquarks in the antiproton. Therefore, $A_{F B}$ is measured under the assumption that the quarks originate form the proton, and the antiquarks originate from the antiproton (first term in Eq. 6).

Since $q(x)$ in the proton is equal to $\bar{q}(x)$ in the antiproton, the dilepton production cross section can be expressed as follows:

$$
\frac{d \sigma}{d M}(\bar{p} p) \propto \sum_{\text {flavor }} v_{i}\left\{q_{i}\left(x_{1}\right) \cdot q_{i}\left(x_{2}\right)+\bar{q}_{i}\left(x_{1}\right) \cdot \bar{q}_{i}\left(x_{2}\right)\right\}
$$

Here $q_{i}(x)$ denote the quark distributions $(u(x), d(x)$, $s(x), c(x), b(x))$ and $\bar{q}_{i}(x)$ denotes the antiquark distributions $(\bar{u}(x), \bar{d}(x), \bar{s}(x), \bar{c}(x), \bar{b}(x))$ in the nucleon. The parameters $v_{i}$ denote the $Z / \gamma$ couplings for each flavor. Here, $v_{i}$ are functions of both the dilepton mass and $\sin ^{2} \theta_{\text {eff }}^{\text {lept }}$.

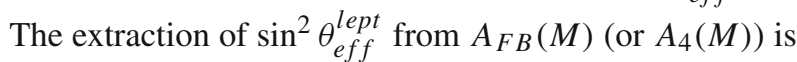
sensitive to PDFs for two reasons. First, $A_{F B}(M)$ for charge 2/3 (u-type) quarks and charge 1/3 (d-type) quarks is different. Fig. 1 shows the contributions of u-type quarks (blue), d-type quarks (red) and the sum of the two contributions (black) to $A_{F B}(M)$ at the Tevatron as given by

$A_{F B}^{d-\text { type }} \approx \frac{(d d)_{F}-(d d)_{B}}{(d d)_{F}+(d d)_{B}+(u u)_{F}+(u u)_{B}}$
$A_{F B}^{u-t y p e} \approx \frac{(u u)_{F}-(u u)_{B}}{(d d)_{F}+(d d)_{B}+(u u)_{F}+(u u)_{B}}$

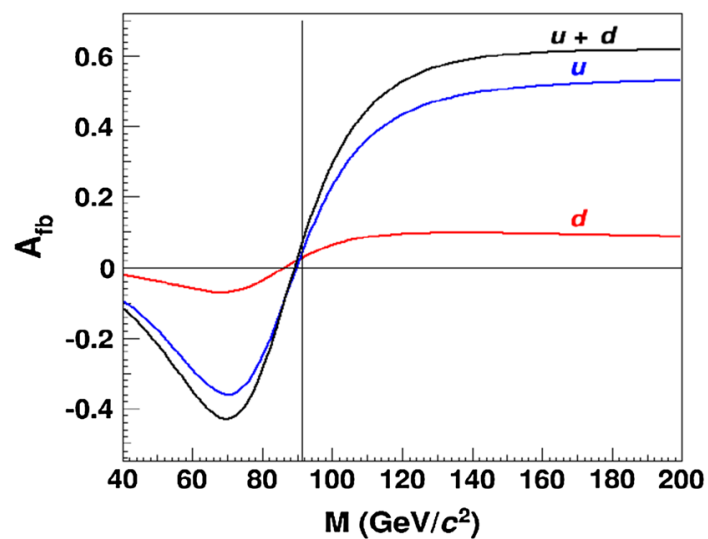

Fig. 1 The contributions of u-type quarks (blue) and d-type quarks (red) to $A_{F B}(M)$ at the Tevatron 
The measured asymmetry is sensitive to the fraction of down quarks in the proton because the asymmetries for up and down quarks are different. The sensitivity is proportional to

$D_{A F B}^{T e v}(d) \underset{\sim}{\propto} \frac{d\left(x_{1}\right) d\left(x_{2}\right)}{u\left(x_{1}\right) u\left(x_{2}\right)}=\left[\frac{d}{u}\left(x_{1}\right)\right]\left[\frac{d}{u}\left(x_{2}\right)\right]$.

In addition, there is a small fraction of events for which the annihilation is between sea antiquarks in the proton with a sea quarks in the antiproton (second term in Eq. 6). The forwardbackward asymmetry $A_{F B}(M)$ of the second term in Eq. 6 is opposite to the $A_{F B}(M)$ of the larger first term. This also results in a dilution $\left(D_{A F B}^{T e v}(\bar{q})\right)$ of the measured asymmetry.

$D_{A F B}^{T e v}(\bar{q}) \propto \frac{\sum_{\text {flavor }} v_{q} \bar{q}\left(x_{1}\right) \cdot \bar{q}\left(x_{2}\right)}{u\left(x_{1}\right) u\left(x_{2}\right)}$

The antiquark dilution is primarily from $u$ type antiquarks. For proton-antiproton collisions, most of the cross section is near $y=0\left(x_{1} \approx x_{2}\right)$. Therefore, the PDF uncertainty in the extraction of $\sin ^{2} \theta_{\text {eff }}^{\text {lept }}$ from $A_{F B}(M)$ (or $A_{4}(M)$ ) at the Tevatron depends primarily on how well we can constrain the following contributions to the dilution at $x_{1}=M_{z} / \sqrt{s}$.

$D_{A F B}^{T e v}(d) \stackrel{d}{\sim}\left[\frac{d}{u}\left(x_{1}\right)\right]^{2}$

$\left.D_{A F B}^{T e v}(\bar{q}) \stackrel{\bar{u}}{\sim}\left(x_{1}\right)\right]^{2}$

\subsection{W charge asymmetry at the Tevatron}

The $W^{-} / W^{+}$ratio at the Tevatron can be written as

$\left(\frac{W^{-}}{W^{+}}\right)^{T e v} \approx \frac{d\left(x_{1}\right) u\left(x_{2}\right)+s\left(x_{1}\right) c\left(x_{2}\right)}{u\left(x_{1}\right) d\left(x_{2}\right)+c\left(x_{1}\right) s\left(x_{2}\right)} \approx \frac{d}{u}\left(x_{1}\right) / \frac{d}{u}\left(x_{2}\right)$

Precise measurements of the $W$ asymmetry provide information on the $d / u$ ratio at the Tevatron. These measurements are important to constrain the PDF uncertainties for the direct measurement of the $\mathrm{W}$ mass. However, at the Tevatron these measurements do not provide information relevant to the measurement of $\sin ^{2} \theta_{\text {eff } f}$ for two reasons. First, there is no information at $y=0\left(x_{1} \approx x_{2}\right)$ since here the $W$ charge asymmetry at the Tevatron is zero. Secondly, at the Tevatron, the $W$ charge asymmetry does not provide information on the absolute level of $\frac{d}{u}(x)$. The $W$ charge asymmetry at the Tevatron provides information only on the slope of $\frac{d}{u}(x)$ as a function of $x$.

\subsection{PDF uncertainties: Hessian and Replica PDFs}

All PDF groups provide a default (central) PDF set. There are two methods that are used for the determination of PDF uncertainties. The first method is to provide a set of eigenvector error PDFs (Hessian method). The PDF uncertainties in a measurement are determined by repeating the analysis for all of the error PDF sets, and adding in quadrature the difference in the results obtained with the error PDFs and the results obtained with the default PDF.

The second method (which is referred to as replica PDFs) is to provide a set of N (e.g. 100 or 1000) replica PDFs. Each of the PDF replicas has equal probability of being correct. The central value of any observable is the average of the values $s_{i}=\left(\sin ^{2} \theta_{W}\right)_{i}$ extracted with each one of the $\mathrm{N}$ PDF replicas. The PDF uncertainty $\left(=\sigma_{p d f}\right)$ is the RMS of the values extracted using all $\mathrm{N}$ replicas.

$$
\langle s\rangle=\frac{1}{N} \sum_{i=1}^{N} s_{i}
$$

$\sigma_{p d f}=\sqrt{\frac{\sum_{i=1}^{N}\left(s_{i}-\langle s\rangle\right)^{2}}{N-1}}$

and the uncertainty in the estimate of the PDF uncertainty is $\Delta \sigma_{p d f}=\frac{\sigma_{p d f}}{\sqrt{2(N-1)}}$

The two methods provide equivalent information. For any given a set of Hessian eigenvector PDFs there is a prescription to generate $[7,20,21]$ an arbitrary number of PDF replicas.

\subsection{Reducing PDF uncertainties with new data}

The advantage of the PDF replica method is that constraints from new data can easily be incorporated in any analysis by applying different weights for each replica.

Replicas for which the theory predictions are in agreement with the new data are given higher weights, and replicas for which the predictions are in poor agreement are given lower weights. The weights are derived from the $\chi^{2}$ values of the comparison between the new data and theory prediction each of the PDF replicas.

The central value of any observable is the weighted average of the values extracted using each one of the N PDF replicas. The PDF uncertainty is the weighted root mean square (RMS) of the values extracted each of the $\mathrm{N}$ replicas.

The procedure of including constraints from new data was initially proposed by Giele and Keller [22]. They proposed that each of the N PDF replicas be weighted as follows:

$$
\begin{aligned}
w_{i} & =\frac{e^{-\frac{1}{2} \chi_{i}^{2}}}{\sum_{i=1}^{N} e^{-\frac{1}{2} \chi_{i}^{2}}} \\
\langle s\rangle & =\sum_{i=1}^{N} w_{i} s_{i} \\
\sigma_{p d f} & =\sqrt{\frac{\sum_{i=1}^{N} w_{i}\left(s_{i}-\langle s\rangle\right)^{2}}{1-1 / N_{e f f}}}
\end{aligned}
$$


The weights reduce the effective number of replicas from $\mathrm{N}$ to $N_{\text {eff }}$ where

$N_{e f f}=\frac{1}{\sum_{i=1}^{N} w_{i}^{2}}$

and the uncertainty in the estimate of the PDF uncertainty is $\Delta \sigma_{p d f} \approx \frac{\sigma_{p d f}}{\sqrt{2\left(N_{e f f}-1\right)}}$.

More recent discussions of the method can be found in references [20,21,23-25]. In the sections that follow we show how the mass and rapidity dependence of $A_{F B}$ can be used to both provide additional constraints and reduce the PDF uncertainty in measurements of $\sin ^{2} \theta_{W}$.

\subsection{Number of replicas needed}

Typically between 100 and 1000 PDF replicas are used. A large number of replicas is only needed if the new data that is being incorporated is so precise that the number of effective replicas drops below 10. This only happens if the statistical errors of the new data are much smaller than the PDF uncertainties.

For the electroweak measurements that are discussed in this paper the statistical errors which are achievable in the next few years are typically within a factor of 2-3 of the PDF uncertainties. Therefore, 100 replicas are typically sufficient.

\subsection{Mass dependance of $A_{F B}(M)$ as a function of $\sin ^{2} \theta_{W}$ and PDFs at the Tevatron}

The sensitivity of the mass dependence of $A_{F B}(M)$ on $\sin ^{2} \theta_{W}$ and PDFs is different. In the region of the $Z$ pole, $A_{F B}(M)$ is sensitive to the vector couplings, which depend on $\sin ^{2} \theta_{W}$. At higher and lower mass $A_{F B}(M)$ is sensitive to the axial coupling and therefore insensitive to value of $\sin ^{2} \theta_{W}$.

In contrast, the magnitude of the dilution of $A_{F B}(M)$ depends on the PDFs. The sensitivity to PDFs is largest in regions where $A_{F B}(M)$ is large (i.e. away from the $Z$ pole).

Figure 2 shows $A_{F B}(M)$ as a function dilepton mass at the Tevatron for $\sin ^{2} \theta_{W}=0.2244$. The band corresponds to the predicted values of $A_{F B}(M)$ for the default NNPDF 3.0 (NNLO) PDF (261000), and ten NNPDF 3.0 (NNLO) replicas. $A_{F B}(M)$ is shown for $\sqrt{s}=1.96 \mathrm{TeV}$ and dilepton rapidity less 1.7 , which corresponds to a typical acceptance for Tevatron experiments (CDF or D0).

Figure 3a shows the sensitivity of $A_{F B}(M)$ at the Tevatron to PDFs. The lines are the difference between $A_{F B}(M)$ for 10 NNPDF 3.0 (NNLO) replicas and $A_{F B}(M)$ calculated for the central default NNPDF 3.0 (NNLO) (261000). Here $\sin ^{2} \theta_{W}$ is fixed at a value of 0.2244 . The difference originates from

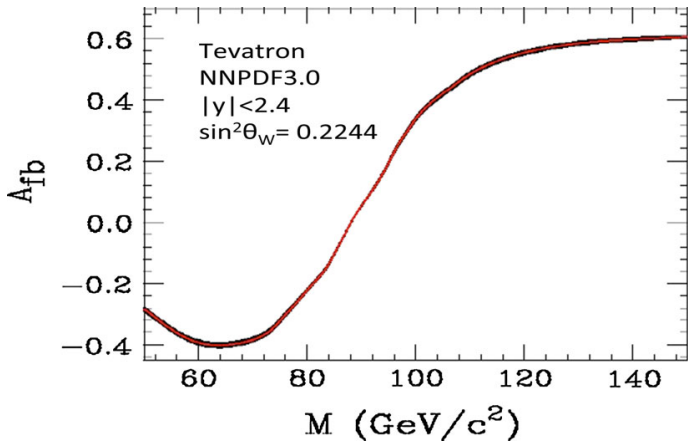

Fig. $2 A_{F B}$ versus dilepton mass at the Tevatron for $\sin ^{2} \theta_{W}=0.2244$ and the default NNPDF 3.0 (NNLO) PDF (261000). The band corresponds to ten NNPDF replicas
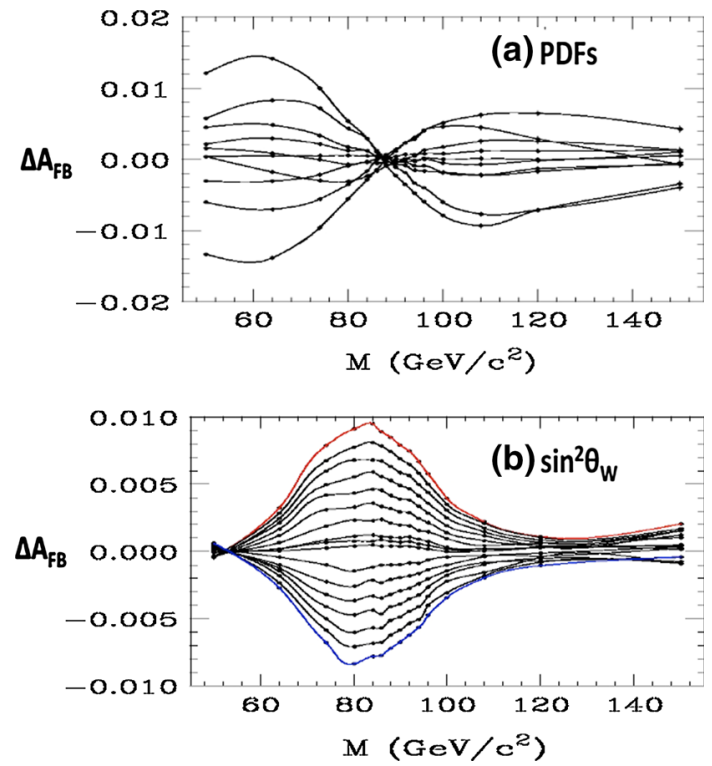

Fig. 3 Tevatron: a The difference between $A_{F B}(M)$ for 10 NNPDF 3.0 (NNLO) replicas and $A_{F B}(M)$ calculated for the default NNPDF 3.0 (NNLO) (261000). Much of the difference originates form the different dilution factors for each of the NNPDF replicas. Here $\sin ^{2} \theta_{W}$ is fixed at a value of 0.2244 . b The difference between $A_{F B}(M)$ for different values of $\sin ^{2} \theta_{W}$ ranging from 0.2220 (shown at the top in red) to 0.2265 (shown on the bottom in blue), and $A_{F B}(M)$ for $\sin ^{2} \theta_{W}=0.2244$. Here $A_{F B}(M)$ is calculated with the default NNPDF 3.0 (NNLO)

the differences in $\frac{d}{u}(x)$ and the antiquark fractions for the different PDF replicas.

Figure $3 \mathrm{~b}$ shows the sensitivity of $A_{F B}(M)$ at the Tevatron to $\sin ^{2} \theta_{W}$. The lines are the difference between the calculated $A_{F B}(M)$ for $\sin ^{2} \theta_{W}$ values ranging from 0.2220 (show at the top in red) to 0.2265 (shown in the bottom in blue) and $A_{F B}(M)$ for $\sin ^{2} \theta_{W}=0.2244$. Here $A_{F B}(M)$ is calculated with the default NNPDF 3.0 (NNLO) (261000).

As shown in Fig. 3a there is a large difference in the $A_{F B}(M)$ predictions for PDF sets with different $\frac{d}{u}(x)$ and antiquark fractions $\frac{\bar{q}}{q}(x)$ in regions where $A_{F B}(M)$ is large and positive $(\mathrm{M}>100 \mathrm{GeV})$. The changes in $A_{F B}(M)$ in 
regions where $A_{F B}(M)$ is large and negative $(\mathrm{M}<80 \mathrm{GeV})$ are in the opposite direction.

In contrast, as shown in Fig. 3b, different values of $\sin ^{2} \theta_{W}$ change $A_{F B}(M)$ primarily in the region near the $Z$ pole. However, here the change is in the same direction above and below the $Z$ pole. Therefore, if we extract $\sin ^{2} \theta_{W}$ from $A_{F B}(M)$ data with different PDFs, PDFs with poor values of $\chi^{2}$ are less likely to be correct.

\subsection{MC studies of dilepton production at Tevatron}

The $10 \mathrm{fb}^{-1}$ Run II $e^{+} e^{-}$data sample at CDF corresponds to about $500 \mathrm{~K}$ events. A similar sample was collected by the D0 experiment [14]. The acceptance of the Tevatron experiments limits the sample to events with dilepton rapidity $|y|<1.7$.

We simulate $A_{F B}(M)$ measurements corresponding a 10 $\mathrm{fb}^{-1}$ statistical sample at the Tevatron with three different input assumptions for $A_{F B}$. In all cases we use $\sin ^{2} \theta_{W}=$ 0.2244 and calculate $A_{F B}$ in 15 bins for dilepton mass spanning the range from $\mathrm{M}=50 \mathrm{GeV}$ to $\mathrm{M}=150 \mathrm{GeV}$. We generate pseudo data for three input assumptions. For each input assumption we generate a set of 1600 pseudo-experiments.

- The input assumption for the first set of 1600 pseudo experiments is that $A_{F B}(M)$ is equal to the predictions of a Tree-level calculation (including EBA EW radiative corrections $[12,13])$ calculated with the default NNPDF 3.0 (NNLO) PDF set.

- The input assumption for the second set of 1600 pseudo experiments is that $A_{F B}(M)$ is equal to the predictions of a Tree-level calculation (including EBA EW radiative corrections $[12,13])$ calculated with the default NNPDF 2.3 (NNLO) PDF set.

- The input assumption for the third set of 1600 pseudo experiments is that $A_{F B}(M)$ is equal to the predictions of RESBOS [18] (modified to include EBA EW radiative corrections $[12,13])$ calculated with the CTEQ 6.6 PDF set.

\subsubsection{Tevatron pseudo data: default NNPDF 3.0 (NNLO) and default NNPDF 2.3 (NNLO)}

For the first set of 1600 pseudo experiments the default NNPDF 3.0 (NNLO) is used to generate pseudo data. The simulated values of $A_{F B}(M)$ for each experiment are compared to $A_{F B}(M)$ templates generated at Tree-level for a range of values of $\sin ^{2} \theta_{W}$ for each of the 100 NNPDF 3.0 (NNLO) PDF replicas. For each replica we extract the best fit value of $\sin ^{2} \theta_{W}$, the corresponding statistical error and the fit $\chi_{A f b}^{2}$. There are about $500 \mathrm{~K}$ dimuon events in each Tevatron pseudo-experiment, which results in a statistical error in $\sin ^{2} \theta_{W}$ of \pm 0.00042 ,

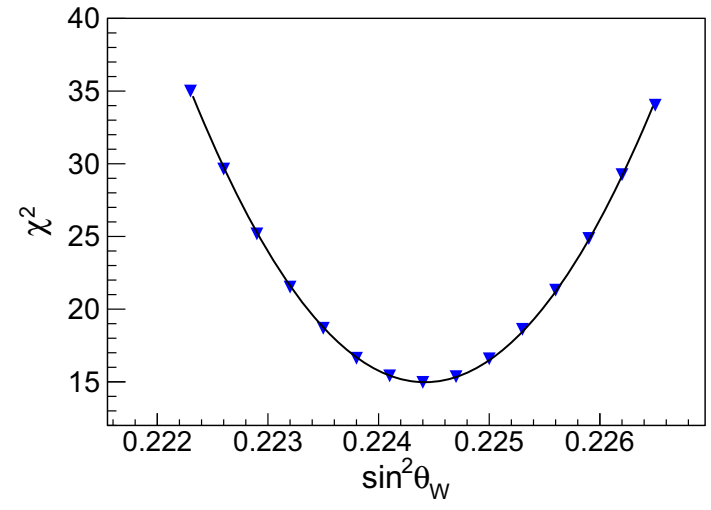

Fig. 4 An example of the extraction of $\sin ^{2} \theta_{W}$ from $A_{F B}(M)$ data at the Tevatron. Here, $\chi_{A f b}^{2}$ is plotted for different values of $\sin ^{2} \theta_{W}$. The extracted value of $\sin ^{2} \theta_{W}$ is the value with the minimum $\chi_{A f b}^{2}$ and the statistical error corresponds to a change of $\chi_{A f b}^{2}$ by \pm 1

An example of the extraction of $\sin ^{2} \theta_{W}$ from $A_{F B}(M)$ data at the Tevatron is shown in Fig. 4. Here, $\chi_{A f b}^{2}$ is plotted for different values of $\sin ^{2} \theta_{W}$. The extracted value of $\sin ^{2} \theta_{W}$ is the value with the minimum $\chi_{A f b}^{2}$ and the statistical error corresponds to a change of $\chi_{A f b}^{2}$ by \pm 1 .

For the second set of 1600 pseudo experiments the default NNPDF 2.3 (NNLO) is used to generate pseudo data and the extraction of $\sin ^{2} \theta_{W}$ is done using 100 NNPDF 2.3 (NNLO) PDF replicas.

For each set, the extracted value of $\sin ^{2} \theta_{W}$ and the PDF uncertainty are done in two ways.

1. The standard average and RMS of the $\sin ^{2} \theta_{W}$ values for the 100 PDF replicas.

2. The $\chi_{A f b}^{2}$ weighted average and weighted RMS of the $\sin ^{2} \theta_{W}$ values for the 100 PDF replicas.

For each of the 100 NNPDF 3.0 (NNLO) (or NNPDF 2.3 (NNLO)) replicas we calculate the average of the 1600 extracted values of $\sin ^{2} \theta_{W}$, the average of the $1600 \mathrm{PDF}$ uncertainties, and the average 1600 statistical errors. These average quantities have small fluctuation and represent the result of one pseudo experiment on average. The average of the 1600 PDF uncertainties is an estimate of the typical uncertainty for one individual pseudo experiment. In order to test for possible bias in the method, the average of the 1600 extracted values of $\sin ^{2} \theta_{W}$ is compared the 0.22420 , which is the value used in the generation.

As expected in both analyses the average extracted value of $\sin ^{2} \theta_{W}$ is the same as the value with which the pseudo data has been generated $(0.2242)$, as shown in Table 1 . With the $\chi_{A f b}^{2}$ weighting method the PDF uncertainty in the extracted value of $\sin ^{2} \theta_{W}$ is reduced from \pm 0.00027 to \pm 0.00020 . This illustrates that although the statistical error in $\sin ^{2} \theta_{W}$ 
Table 1 Values of $\sin ^{2} \theta_{W}$ with statistical errors and PDF uncertainties expected at the Tevatron for a $10 \mathrm{fb}^{-1}$ sample for a CDF like detector. The PDF uncertainty for a standard analysis is compared to the PDF uncertainty for an analysis with $\chi_{A f b}^{2}$ weighting. The default NNPDF 3.0 (NNLO) is used to generate the pseudo data in the first column and the default NNPDF 2.3 (NNLO) is used to generate the pseudo data in the second column. All pseudo data are generated with $\sin ^{2} \theta_{W}=0.22420$

\begin{tabular}{lll}
\hline & Input & Input \\
CDF-like detector & Tree-level & Tree-level \\
Pseudo-experiment & Default & Default \\
Tevatron $10 \mathrm{fb}^{-1}$ & NNPDF 3.0 & NNPDF 2.3 \\
$500 K$ reconstructed & $(261000)$ & $(261000)$ \\
$e^{+} e^{-}$events & $($NNLO) & $($NNLO) \\
\hline $\sin ^{2} \theta_{W}$ input & 0.22420 & 0.22420 \\
\hline Statistical error & \pm 0.00042 & \pm 0.00042 \\
$\Delta$ sin $^{2} \theta_{W}$ & & \\
CT10 PDF error & \pm 0.00026 & \pm 0.00026 \\
\hline Number of analysis replicas & 100 & 100 \\
NNPDF replica set & NNPDF 3.0 & NNPDF 2.3 \\
Templates & Tree-level & Tree-level \\
Average method & $N_{\text {eff }}=100$ & $N_{\text {eff }}=100$ \\
Extracted sin & \\
PDF error RMS & 0.22420 & 0.22420 \\
(uncertainty in PDF error) & \pm 0.00027 & \pm 0.00028 \\
$\chi_{A}^{2}$ weighting & $(0.00002)$ & $(0.00002)$ \\
extracted $\sin ^{2} \theta_{W}$ & $N_{\text {eff }}=88$ & $N_{\text {eff }}=85$ \\
$P D F$ error weighted & 0.22420 & 0.22420 \\
(uncertainty in PDF error) & \pm 0.00020 & \pm 0.00022 \\
\hline & $(0.00002)$ & $(0.00002)$ \\
\hline
\end{tabular}

of \pm 0.00042 is somewhat larger than the PDF uncertainty of \pm 0.00027 , the $A_{F B}$ data at higher and lower mass has sufficient precision to constrain the PDFs which yields a $25 \%$ reduction in the PDF uncertainty.

A graphical illustration of the method is shown in Fig. 5a and $b$. For each PDF replica, we calculate the average of the extracted values of $\sin ^{2} \theta_{W}$ and the average $\chi_{A f b}^{2}$ of the fits for the 1600 pseudo experiments. Figure $5 \mathrm{a}$ and $\mathrm{b}$ show the scatter plot of the average of the extracted values of $\sin ^{2} \theta_{W}$ and the average $\chi_{A f b}^{2}$ for the 100 PDF replicas.

Also shown on the plot is the input value of $\sin ^{2} \theta_{W}$ with the average statistical error of one pseudo experiment. In addition, we show the average of the extracted values $\sin ^{2} \theta_{W}$ and average PDF uncertainty for both the standard analysis, and the $\chi_{A f b}^{2}$ weighted analysis.

\subsubsection{Pseudo data: RESBOS with CTEQ 6.6 PDF set}

We perform two analyses of the third set of 1600 pseudo experiments (CTEQ 6.6 pseudo data). In one analysis the simu-
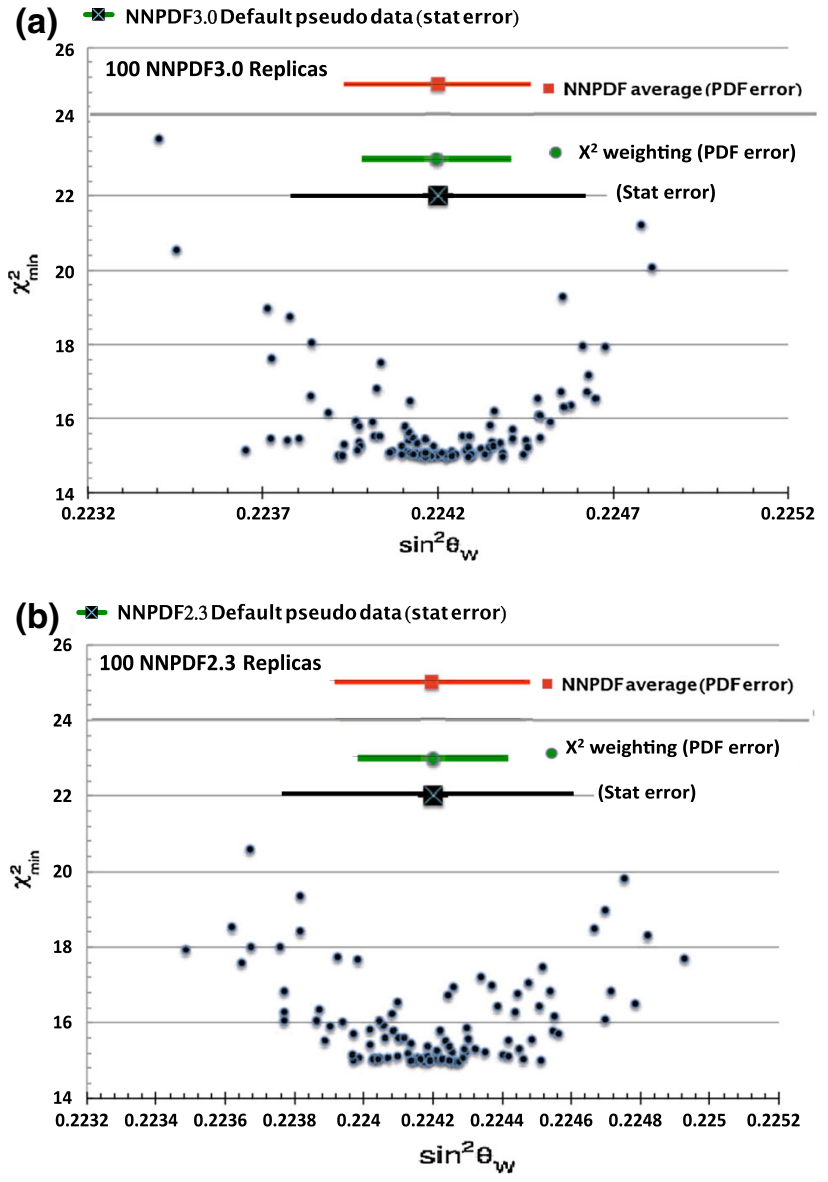

Fig. 5 Tevatron: a graphical illustration of the analysis of one typical pseudo experiment. Shown is a scatter plot of $\sin ^{2} \theta_{W}$ and $\chi_{A f b}^{2}$ values for 100 PDF replicas. a For pseudo experiment generated with the default NNPDF 3.0 (NNLO) and $\sin ^{2} \theta_{W}=0.22420$ at Tree-level. $\mathbf{b}$ or pseudo experiment generated with the default NNPDF 2.3 (NNLO) and $\sin ^{2} \theta_{W}=0.22420$ at Tree-level. Also shown on the plot is the input value of $\sin ^{2} \theta_{W}$ with the average statistical error of one pseudo experiment. In addition, we show the average of the extracted values $\sin ^{2} \theta_{W}$ and average PDF uncertainty for both the standard analysis, and the $\chi_{A f b}^{2}$ weighted analysis

lated values of $A_{F B}(M)$ for each experiment are compared to templates calculated at Tree-level for each of the $100 \mathrm{NNPDF}$ 3.0 (NNLO) PDF replicas. In the other analysis the simulated values of $A_{F B}(M)$ for each experiment are compared to templates calculated at Tree-level for each of the 100 NNPDF 2.3 (NNLO) PDF replicas. In each of the two analyses, $\sin ^{2} \theta_{W}$ is extracted using both the standard average and RMS, and also the $\chi_{A f b}^{2}$ weighted average and RMS of the 100 PDF replicas. The results are summarized in Table 2.

In the analysis of the RESBOS/CTEQ 6.6 pseudo data with NNPDF 3.0 (NNLO) replica templates we find that the PDF uncertainty in the extracted value of $\sin ^{2} \theta_{W}$ when we use the standard average is \pm 0.00027 . The PDF uncertainty is reduced to \pm 0.00020 when the $\chi_{A f b}^{2}$ weighting method is used, as shown in Table 2 and Fig. 6 . The effective num- 
Table 2 Tevatron Pseudo data (500K reconstructed $e^{+} e^{-}$events (corresponding to $10 \mathrm{fb}^{-1}$ ). The events are generated with RESBOS and CTEQ 6.6 PDFs for a CDF like detector. Here, we compare $\sin ^{2} \theta_{W}$ values with statistical errors and PDF uncertainties extracted with NNPDF 3.0 (NNLO) PDF replicas and with NNPDF 2.3 (NNLO) PDF replicas. The values of $\sin ^{2} \theta_{W}$ extracted with NNPDF 3.0 (NNLO) PDF and NNPDF 2.3 (NNLO) are different (for details see text)

\begin{tabular}{lll}
\hline $\sin ^{2} \theta_{W}$ input & 0.22420 & 0.22420 \\
\hline Statistical error $\Delta \sin ^{2} \theta_{W}$ & \pm 0.00042 & \pm 0.00042 \\
CT10 PDF error & \pm 0.00026 & \pm 0.00026 \\
\hline Number of analysis replicas & 100 & 100 \\
NNPDF replica set & NNPDF 3.0 & NNPDF 2.3 \\
Templates & Tree-level & Tree-level \\
Average method & $N_{\text {eff }}=100$ & $N_{\text {eff }}=100$ \\
extracted $\sin ^{2} \theta_{W}$ & 0.22425 & 0.22469 \\
bias & +0.00005 & +0.00049 \\
PDF error RMS & \pm 0.00027 & \pm 0.00027 \\
(uncertainty in PDF error) & $(0.00002)$ & $(0.00002)$ \\
$A_{F B} \chi_{A f b}^{2}$ weighting & $N_{e f f}=88$ & $N_{\text {eff }}=63$ \\
extracted $\sin ^{2} \theta_{W}$ & 0.22425 & 0.22452 \\
bias & +0.00005 & +0.00032 \\
PDF error weighted & \pm 0.00020 & \pm 0.00021 \\
(uncertainty in PDF error) & $(0.00002)$ & $(0.00002)$ \\
\hline
\end{tabular}

ber of replicas is reduced from 100 to 88 . The average value is $\sin ^{2} \theta_{W}=0.22425$ for both the standard analysis and the $\chi_{A f b}^{2}$ weighting analysis. The very small difference $(+0.00005)$ from the input value of $\sin ^{2} \theta_{W}=0.22420$ is attributed to the difference between the RESBOS pseudo data which is generated at NLO and the templates which were done at LO Tree-level.

In contrast, the standard analysis with the NNPDF 2.3 (NNLO) replica templates yields a value which is biased by $+0.00049 \pm 0.00001$. This is larger than the PDF uncertainty of \pm 0.00027 . This bias indicates that the NNPDF 2.3 (NNLO) set is not fully consistent with the CTEQ 6.6 PDF for the Bjorken $x$ region for the production of $\mathrm{Z}$ bosons at the Tevatron. When the $\chi_{A f b}^{2}$ weighting technique is used instead, the bias is partially reduced from $+0.00049 \pm 0.00001$ to $+0.00032 \pm 0.00001$, and the effective number of PDFs is reduced from 100 to 63 . The reduced bias is expected because $\chi_{A f b}^{2}$ weighting assigns small weights to a fraction of NNPDF 2.3 (NNLO) PDF replicas which are incompatible with the CTEQ 6.6. pseudo data.

As shown in Fig. 6 the distribution of $\chi_{A f b}^{2}$ values versus $\sin ^{2} \theta_{W}$ provides a powerful tool to discriminate against PDF sets which are incompatible with each other or with the data. Our study indicates that CTEQ 6.6 PDFs are inconsistent with the NNPDF 2.3 (NNLO) set, but are consistent with the NNPDF 3.0 (NNLO) set. One of the difference between NNPDF 3.0 and
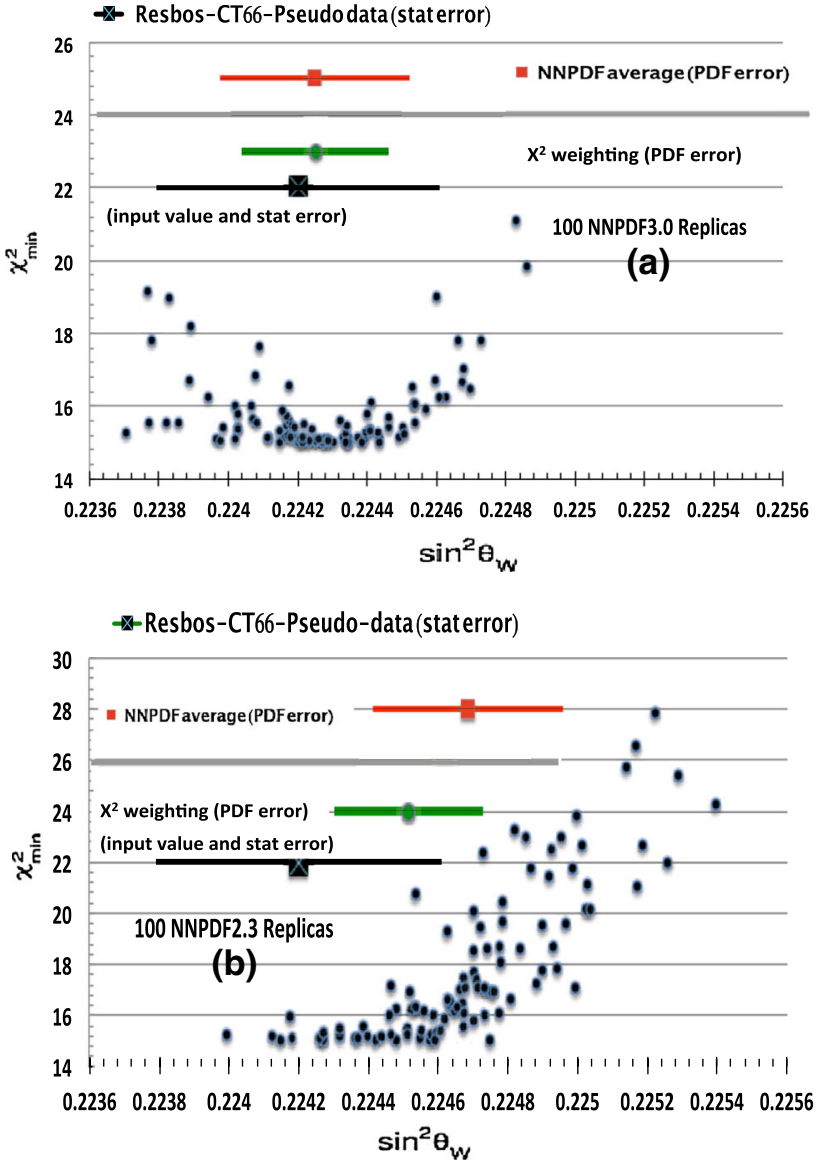

Fig. 6 Analysis of a Tevatron pseudo-experiment. The pseudo data are generated by RESBOS with CTEQ 6.6 PDF and $\sin ^{2} \theta_{W}=0.22420$. This figure illustrates that with the $\chi_{A f b}^{2}$ weighting method we can determine that pseudo data generated with CTEQ 6.6 PDFs are not consistent with the NNPDF 2.3 (NNLO) set. a Analysis with 100 NNPDF 3.0 (NNLO) replicas. b Analysis with 100 NNPDF 2.3 (NNLO) replicas. The distribution of $\chi_{A f b}^{2}$ values versus $\sin ^{2} \theta_{W}$ provides a powerful tool to discriminate against PDF sets which are incompatible with the data. The PDF sets which are compatible with the data should have a symmetric distribution of $\chi_{A f b}^{2}$ values versus $\sin ^{2} \theta_{W}$

NNPDF 2.3 is that NNPDF 2.3 used $W$ asymmetry data which is now known to be incorrect.

\section{Production of dilepton events at the LHC}

At the LHC, dileptons are produced by annihilation of quarks in one proton with antiquarks in the other proton.

$\frac{d \sigma}{d M d y}(p p) \propto \sum_{\text {flavor }} v_{i}\left\{q_{i}\left(x_{1}\right) \bar{q}_{i}\left(x_{2}\right)+\bar{q}_{i}\left(x_{1}\right) q_{i}\left(x_{2}\right)\right\}$

Because on average, quarks carry more momentum than antiquarks, the quark direction is assumed to be the direction of motion of the dilepton pair. This is more likely to be true for dileptons produced at high rapidity. At the LHC 
the asymmetry from the first term of Eq. 18 is diluted by the asymmetry of the second term (which is in the opposite direction). Equation 18 shows that for $y=0\left(x_{1}=x_{2}\right)$ the asymmetries for the two terms cancel each other.

An estimate of the dilution of $A_{F B}(M)$ can be obtained from the probability to misidentify the direction of the quark $f(M, y)$. For $p p$ collisions $f(M, y)$ is the fraction of events for which the antiquark carries more momentum than the quark.

$$
f(M, y) \approx \frac{\sum_{\text {flavor }} v_{i}\left\{\bar{q}_{i}\left(x_{1}\right) q_{i}\left(x_{2}\right)\right\}}{\sum_{\text {flavor }} v_{i}\left\{q_{i}\left(x_{1}\right) \bar{q}_{i}\left(x_{2}\right)+\bar{q}_{i}\left(x_{1}\right) q_{i}\left(x_{2}\right)\right\}}
$$

The asymmetry is significant only when $x_{1}$ is large and $x_{2}$ is small (when $x_{2}$ is small, $u\left(x_{2}\right) \approx d\left(x_{2}\right) \approx \bar{u}\left(x_{2}\right) \approx \bar{d}\left(x_{2}\right)$ ). The asymmetry for $u$ quarks dominates, and the fractions of $d$ quarks and $\bar{u}$ antiquarks are sources of dilution.

$D_{A F B}^{L H C}(d) \stackrel{d\left(x_{1}\right) \bar{d}\left(x_{2}\right)}{\sim} \approx \frac{d}{u\left(x_{1}\right) \bar{u}\left(x_{2}\right)}\left(x_{1}\right)$

$D_{A F B}^{L H C}(\bar{q}) \underset{\sim}{\propto} \frac{\bar{u}\left(x_{1}\right) u\left(x_{2}\right)}{u\left(x_{1}\right) \bar{u}\left(x_{2}\right)} \approx \frac{\bar{u}}{u}\left(x_{1}\right)$

Since $x_{1}=\frac{M}{\sqrt{s}} e^{+y}$ both the mass and rapidity dependence of $A_{F B}$ provides information on PDFs.

At the LHC, the $\mathrm{W}$ asymmetry also provides information on the $\mathrm{d} / \mathrm{u}$ ratio. The $W^{-} / W^{+}$ratio at the $\mathrm{LHC}$ can be written as

$$
\begin{aligned}
\left(\frac{W^{-}}{W^{+}}\right)^{L H C} & \approx \frac{d\left(x_{1}\right) \bar{u}\left(x_{2}\right)+s\left(x_{1}\right) \bar{c}\left(x_{2}\right)}{u\left(x_{1}\right) \bar{d}\left(x_{2}\right)+c\left(x_{1}\right) \bar{s}\left(x_{2}\right)} \\
& \approx \frac{d / u\left(x_{1}\right)}{\bar{d} / \bar{u}\left(x_{2}\right)} \approx \frac{d}{u}\left(x_{1}\right)
\end{aligned}
$$

Unlike the situation at the Tevatron, more precise $\mathrm{W}$ asymmetry measurements at the LHC provide information on the absolute value of $\frac{d}{u}\left(x_{1}\right)$. Therefore, new measurements of the $\mathrm{W}$ charge asymmetry at the LHC (which have not yet been incorporated into PDF fits) can be used in combination with the constraints from $A_{F B}$ to reduce the PDF uncertainty in the extractions of $\sin ^{2} \theta_{\text {eff }}$ and $\sin ^{2} \theta_{W}$ at the LHC.

Combining constraints from both $A_{F B}$ and new W asymmetry measurements can be done by adding the values of $\chi_{\text {Wasym }}^{2}$ from the comparison of the new W asymmetry data with the predicted W asymmetry for each PDF replica, to the $\chi_{A f b}^{2}$ values from the fits to extract $\sin ^{2} \theta_{\text {eff }}$ from the $A_{F B}(M, y)$ data for each PDF replica.

4.1 Mass dependance of $A_{F B}(M, y)$ as a function of $\sin ^{2} \theta_{\text {eff }}$ and PDFs at the LHC

The top panel of Fig. 7 shows $A_{F B}(M, y)$ at the LHC at $\sqrt{s}=$ $8 \mathrm{TeV}$ for six rapidity bins $0<|y|<0.4,0.4<|y|<0.8$, $0.8<|y|<1.2,1.2<|y|<1.6,1.6<|y|<1.0$ and

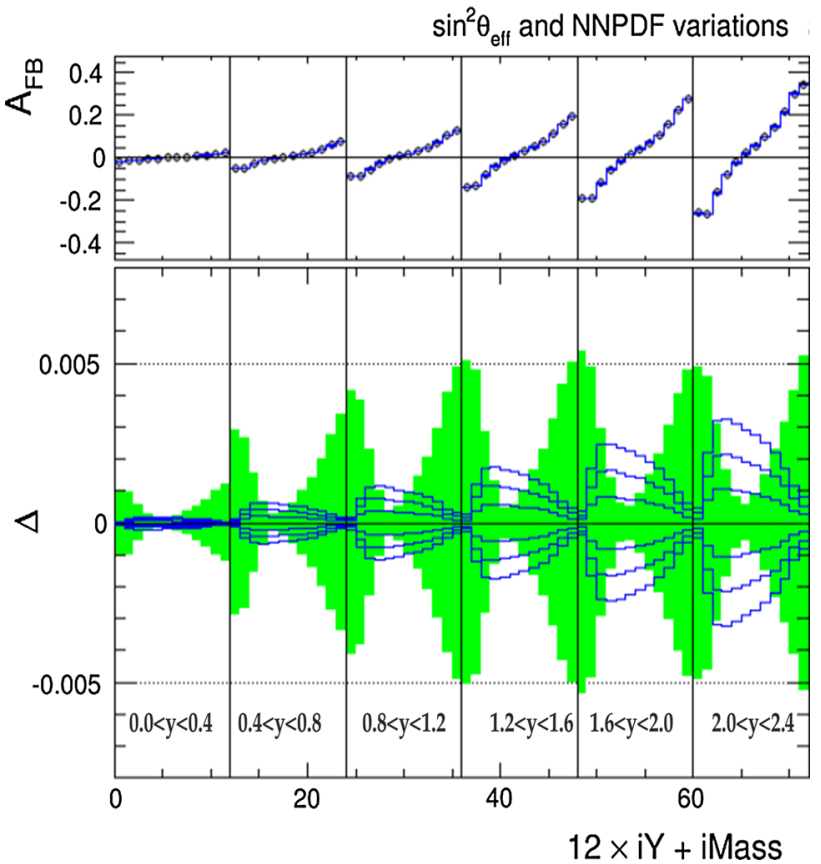

Fig. 7 LHC: top panel $A_{F B}$ at the LHC at $\sqrt{s}=8 \mathrm{TeV}$ for six rapidity bins (iY $=0-5)$ with average $|y|$ values of $0.2,0.6,1.0,1.4,1.8$ and 2.2. For each rapidity bin there are twelve mass bins discussed in the text (iMass $=0-11$ ). The horizontal scale for each of the six plots is the dimuon invariant mass for each rapidity bin expressed as $12 \times i Y+i$ Mass. Bottom panel the green bands span the difference between $A_{F B}(M)$ calculated for the 100 NNPDF 3.0 (NLO) replicas and $A_{F B}(M)$ calculated for the central default NNPDF 3.0 (NLO) for the six dimuon rapidity bins. The blue lines are the differences between $A_{F B}(M)$ calculated with different values of $\sin ^{2} \theta_{\text {eff }}(0.23120$ $\pm 0.00040, \pm 0.00080$ and \pm 0.00120$)$. and the values calculated with nominal $\sin ^{2} \theta_{\text {eff }}=0.23120$. For all of the blue lines, $A_{F B}(M)$ is calculated with the central default NNPDF 3.0 (NLO). The calculations are done with the POWHEG MC generator

$2.0<|y|<2.4(\mathrm{iY}=0-5)$. These six bins have average $|y|$ values of $0.2,0.6,1.0,1.4,1.8$ and 2.2. The mass bins are 60-70, 70-78, 78-84, 84-87, 87-89, 89-91, 91-93, 93-95, 95-98, 98-104, 104-112 and 112-120 GeV. The horizontal scale for each of the six plots is the dimuon invariant mass for each rapidity bin expressed as $12 \times i Y+i$ Mass.

The calculations are done with the POWHEG [19] MC generator. The version of POWHEG that is used does not include electroweak radiative corrections. Therefore, this version of POWHEG requires an input value of $\sin ^{2} \theta_{e f f}$ for the calculation of $A_{F B}$

The green bands in the bottom panel of Fig. 7 span the difference between $A_{F B}(M, y)$ calculated with the $100 \mathrm{NNPDF}$ 3.0 (NLO) replicas and $A_{F B}(M, y)$ calculated with the default NNPDF 3.0 (NLO) PDF.

The blue lines are the differences between $A_{F B}(M, y)$ calculated for several values of $\sin ^{2} \theta_{\text {eff }}\left(\sin ^{2} \theta_{\text {eff }}=\right.$ $0.23120 \pm 0.00040, \pm 0.00080$ and \pm 0.00120$)$ and $A_{F B}$ $(M, y)$ for the nominal $\sin ^{2} \theta_{\text {eff }}=0.23120$. For all of the blue lines, $A_{F B}(M, y)$ is calculated with the default NNPDF 3.0(NLO) PDF. 
As is the case for the Tevatron, the dependence of $A_{F B}(M, y)$ on $\sin ^{2} \theta_{e f f}$ and on PDFs is different. In the region of the $Z$ pole, $A_{F B}(M, y)$ is sensitive to the vector couplings, which are functions of $\sin ^{2} \theta_{\text {eff }}$. At higher and lower mass $A_{F B}(M, y)$ is sensitive to the axial coupling and therefore insensitive to value of $\sin ^{2} \theta_{\text {eff }}$. As is the case for the Tevatron, the magnitude of the dilution of $A_{F B}(M)$ is larger in regions where the absolute value of $A_{F B}(M)$ is large (i.e. away from the $Z$ pole). At the LHC the dilution depends on both $\mathrm{M}$ and $\mathrm{y}$. The combined mass and rapidity dependence of the dilution at the LHC provides more stringent constraints on PDFs than $A_{F B}(M)$ measurements at the Tevatron.

\subsection{MC studies with NNPDF 3.0 PDFs at the LHC}

For studies of $A_{F B}(M, y)$ at the LHC we simulate DrellYan dimuon data for 64 pseudo experiments for a CMS like detector at $\sqrt{s}=8 \mathrm{TeV}$. The pseudo data are generated by the POWHEG NLO MC generator with the default NNPDF 3.0 (NLO) PDFs, The pseudo data are generated with an effective mixing angle $\sin ^{2} \theta_{\text {eff }}=0.23120$.

For each pseudo experiment, we generate a sample of 15.6 Million dimuon events with $M_{\mu \mu}>50 \mathrm{GeV}$, which corresponds to an integrated luminosity of $15.0 \mathrm{fb}^{-1}$. This is similar to the $\approx 19 \mathrm{fb}^{-1}$ of integrated luminosity collected by CMS and ATLAS at $8 \mathrm{TeV}$. We apply acceptance and transverse momentum cuts which are similar to a CMS-like detector. We also smear the muon energy with a muon momentum resolution similar to a CMS-like detector. The final sample consists $6.7 \mathrm{M}$ reconstructed dimuon events.

The $8 \mathrm{TeV} W$ decay lepton asymmetry data at the LHC has not yet been incorporated into the most recent PDF fits. Therefore, in addition to $A_{F B}(M, y)$, we also use the default NNPDF 3.0 (NLO) and generate pseudo data for the $\mathrm{W}$ muon decay asymmetry as a function of muon rapidity (for muon transverse momentum PT $>25 \mathrm{GeV}$ ). This simulates the $\mathrm{W}$ asymmetry measurement at $8 \mathrm{TeV}$.

In the analysis of each of the 64 pseudo experiments generated with the default NNPDF 3.0 (NLO) the extracted values of $A_{F B}(M, y)$ for each experiment are compared to $A_{F B}(M, y)$ templates. The templates are generated with the POWHEG MC for a range of values of $\sin ^{2} \theta_{\text {eff }}$ for each of the $100 \mathrm{NNPDF}$ 3.0 (NLO) PDF replica. For each replica we extract the best fit value of $\sin ^{2} \theta_{e f f}$, the corresponding statistical error and the fit $\chi_{A f b}^{2}$.

In addition, we calculate $\chi_{\text {Wasym }}^{2}$ which is the $\chi^{2}$ for the agreement between the predictions for the $\mathrm{W}$ lepton decay asymmetry and the $\mathrm{W}$ lepton decay asymmetry pseudo data at $8 \mathrm{TeV}$ for each of the $100 \mathrm{PDF}$ replicas.

Figure 8 shows the results from one of the 64 pseudo experiments at the LHC. The top two panels show the
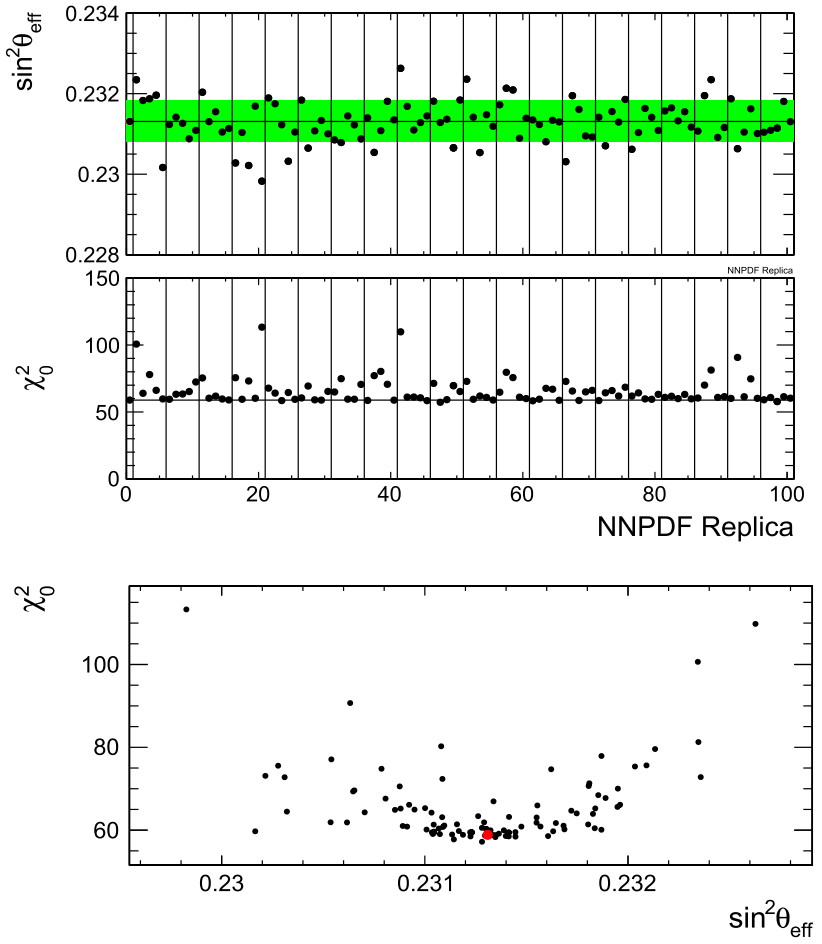

Fig. 8 Analysis of one of the 64 LHC pseudo experiments $(6.7 \mathrm{M}$ dimuon events with CMS-like detector acceptance cuts) with 100 PDF replicas. The pseudo data are generated by the POWHEG MC with the default NNPDF 3.0 (NLO) PDF and $\sin ^{2} \theta_{\text {eff }}=0.23120$. The top two panels show the extracted $\sin ^{2} \theta_{\text {eff }}$ and corresponding $\chi_{A f b}^{2}$ values from fits to $A_{F B}(M, y)$ versus replica number for the 100 NNPDF 3.0 (NLO) replicas. The bottom panel shows the same results in the form of a scatter plot of $\chi_{A f b}^{2}$ values versus $\sin ^{2} \theta_{\text {eff }}$ for one pseudo experiment. The number of degrees of freedom is $71(=6 \times 12-1)$

extracted $\sin ^{2} \theta_{\text {eff }}$ and corresponding $\chi_{A f b}^{2}$ values from fits to $A_{F B}(M, y)$ versus replica number for the 100 NNPDF 3.0 (NLO) replicas. The bottom shows the same results in the form of a scatter plot of $\chi_{A f b}^{2}$ values versus $\sin ^{2} \theta_{e f f}$ for one pseudo experiment. The number of degrees of freedom is 71 $(=6 \times 12-1)$.

For each pseudo experiment we find the mean value and PDF uncertainty of $\sin ^{2} \theta_{\text {eff }}$ from the average and RMS of the $\sin ^{2} \theta_{\text {eff }}$ for the 100 PDF replicas. The average and RMS values are done in three ways:

1. Using the standard average and RMS of the $\sin ^{2} \theta_{\text {eff }}$ fit values. This analysis results in a standard PDF uncertainty of \pm 0.00051 with 100 replicas.

2. Using the $\chi_{A f b}^{2}$ values of the fits to $A_{F B}(M, y)$ to form a weighted average and weighted RMS of the $\sin ^{2} \theta_{\text {eff }}$ values. This analysis results in a PDF uncertainty of \pm 0.00029 with 37 effective replicas.

3. Using the combined $\chi_{A f b}^{2}+\chi_{W a s y m}^{2}$ for the fits to DrellYan $A_{F B}(M, y)$ pseudo data and the fits to the W lep- 
Table 3 Values of $\sin ^{2} \theta_{W}$ with statistical errors and PDF uncertainties expected for a $15 \mathrm{fb}^{-1}$ Drell-Yan dimuon sample at the LHC (at $8 \mathrm{TeV}$ ). The pseudo data are generated by the POWHEG MC generator with the default NNPDF 3.0 (NLO) PDF, and $\sin ^{2} \theta_{\text {eff }}=0.23120$. The sample consists of $6.7 M \mu^{+} \mu^{-}$reconstructed in a CMS like detector. The PDF uncertainty for a standard analysis is compared to the PDF uncertainty for an analysis with both $\chi_{A f b}^{2}$ weighting and $\chi_{A f b}^{2}+\chi_{W \text { asym }}^{2}$ weighting

\begin{tabular}{ll}
\hline $\sin ^{2} \theta_{e f f}$ input & 0.23120 \\
\hline statistical error $\Delta \sin ^{2} \theta_{e f f}$ & \pm 0.00050 \\
CT10 PDF error & \pm 0.00080 \\
\hline Analysis replicas & 100 \\
NNPDF set & NNPDF 3.0 (NLO) \\
Templates & POWHEG \\
Average method & $N_{e f f}=100$ \\
extracted $\sin ^{2} \theta_{e f f}$ & 0.23121 \\
Standard PDF error RMS & \pm 0.00051 \\
(uncertainty in PDF error) & $(0.00004)$ \\
$\chi_{A f b}^{2}$ weighting & $N_{e f f}=37$ \\
extracted $\sin ^{2} \theta_{W}$ & 0.23119 \\
$\chi_{A f b}^{2}$ weighted PDF error RMS & \pm 0.00029 \\
(uncertainty in PDF error) & $(0.00003)$ \\
$\chi_{A f b}^{2}+\chi_{W s y m}^{2}$ weighting & $N_{e f f}=15$ \\
extracted $\sin ^{2} \theta_{W}$ & 0.23122 \\
Weighted PDF error RMS & \pm 0.00026 \\
(uncertainty in PDF error) & $(0.00005)$ \\
\hline
\end{tabular}

ton decay asymmetry pseudo data to form the weighted average and weighted RMS of the $\sin ^{2} \theta_{\text {eff }}$ values. This analysis results in a PDF uncertainty of \pm 0.00026 with 15 effective replicas.

\subsection{Studies with 1000 replicas}

As shown in Table 3, the number of effective PDF replicas is reduced to 15 when we apply constraints from both $\chi_{A f b}^{2}$ and $\chi_{\text {Wasym }}^{2}$. The PDF uncertainty is reduced to \pm 0.00026 . The uncertainty in the estimate of the PDF uncertainty is \pm 0.00005 . If we start with 1000 PDF replicas, the number of effective PDF replicas is $\approx 150$, and the uncertainty in the estimate of the PDF uncertainty is reduced to \pm 0.00002 . Therefore, the analysis is somewhat more robust if we start with 1000 PDF replicas.

Figure 9 shows scatter plots of $\chi_{A f b}^{2}$ values versus $\sin ^{2} \theta_{\text {eff }}$ for one of the 64 LHC pseudo experiments. Here templates are generated with 1000 replicas for (a) NNPDF 3.0(NLO) PDF set (b) CT10(NLO) PDF set, (c) CT14(NLO) PDF set and (d) MMHT(NLO) PDF set. The number of degrees of freedom is $71(=6 \times 12-1)$. The pseudo data are generated with POWHEG with the default NNPDF 3.0 (NLO)
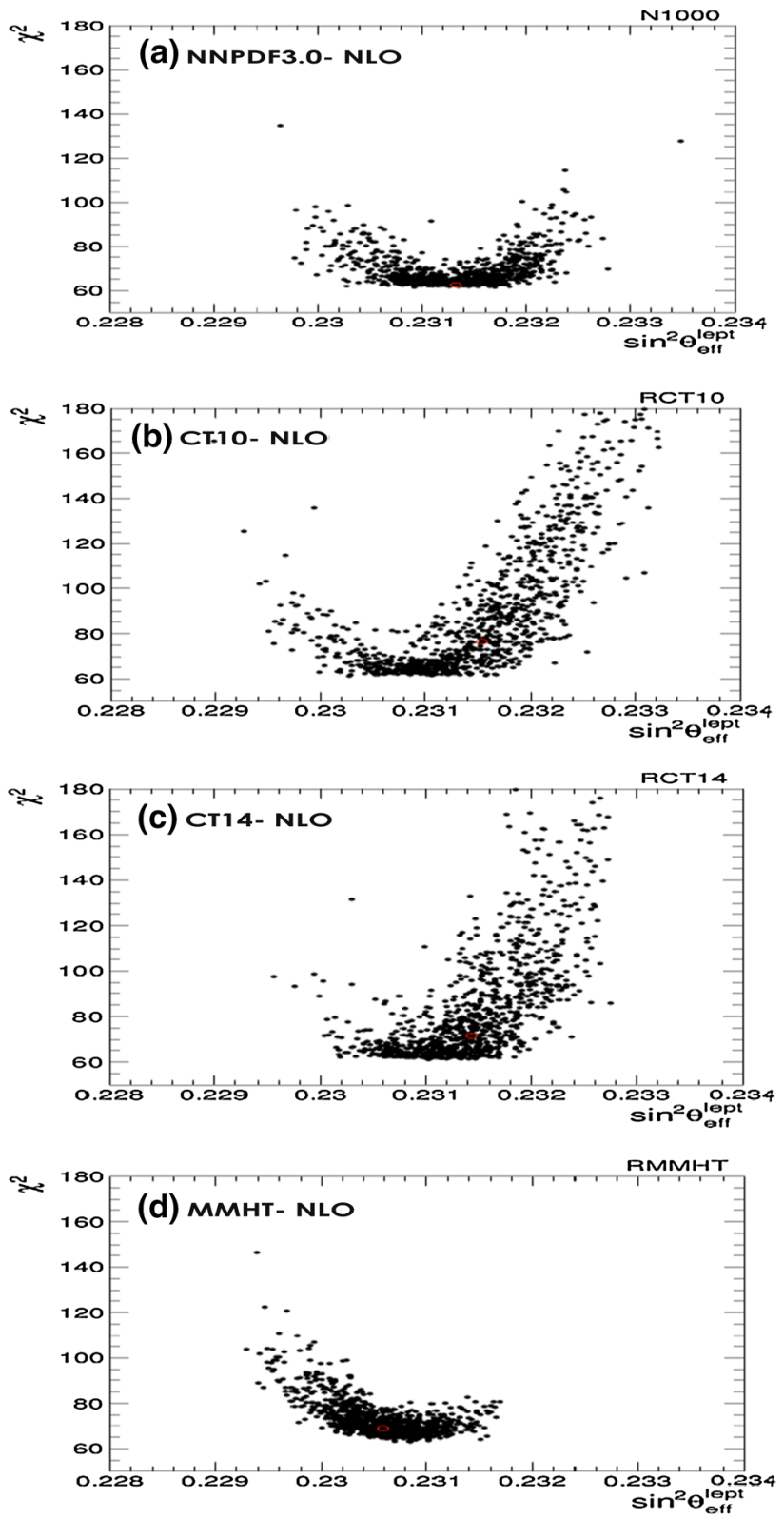

Fig. 9 Scatter plots of $\chi_{A f b}^{2}$ values versus $\sin ^{2} \theta_{\text {eff }}$ for one of the 64 LHC pseudo experiments. Here templates are generated with 1000 replicas for a NNPDF 3.0(NLO), b CT10(NLO), c CT14(NLO), and d MMHT(NLO). The number of degrees of freedom is $71(=6 \times 12-1)$. The pseudo data are generated with POWHEG with the default NNPDF 3.0 (NLO) PDF and $\sin ^{2} \theta_{\text {eff }}=0.23120$ (6.7 M dimuon events with CMS-like detector acceptance cuts)

PDF and $\sin ^{2} \theta_{e f f}=0.23120$. (6.7 dimuon events reconstructed with CMS-like detector acceptance cuts).

In order to reduce the statistical error and investigate the PDF uncertainties, we take the average of 64 pseudo experiments. The statistical error in the average of the 64 $\sin ^{2} \theta_{\text {eff }}$ measurements is $\pm 0.00007(=0.00052 / 8)$. Figure 10 shows the average of the results from the analyses of all 64 LHC pseudo experiments with templates generated with 100 NNPDF3.0 replicas, 1000 NNPDF3.0 replicas, 

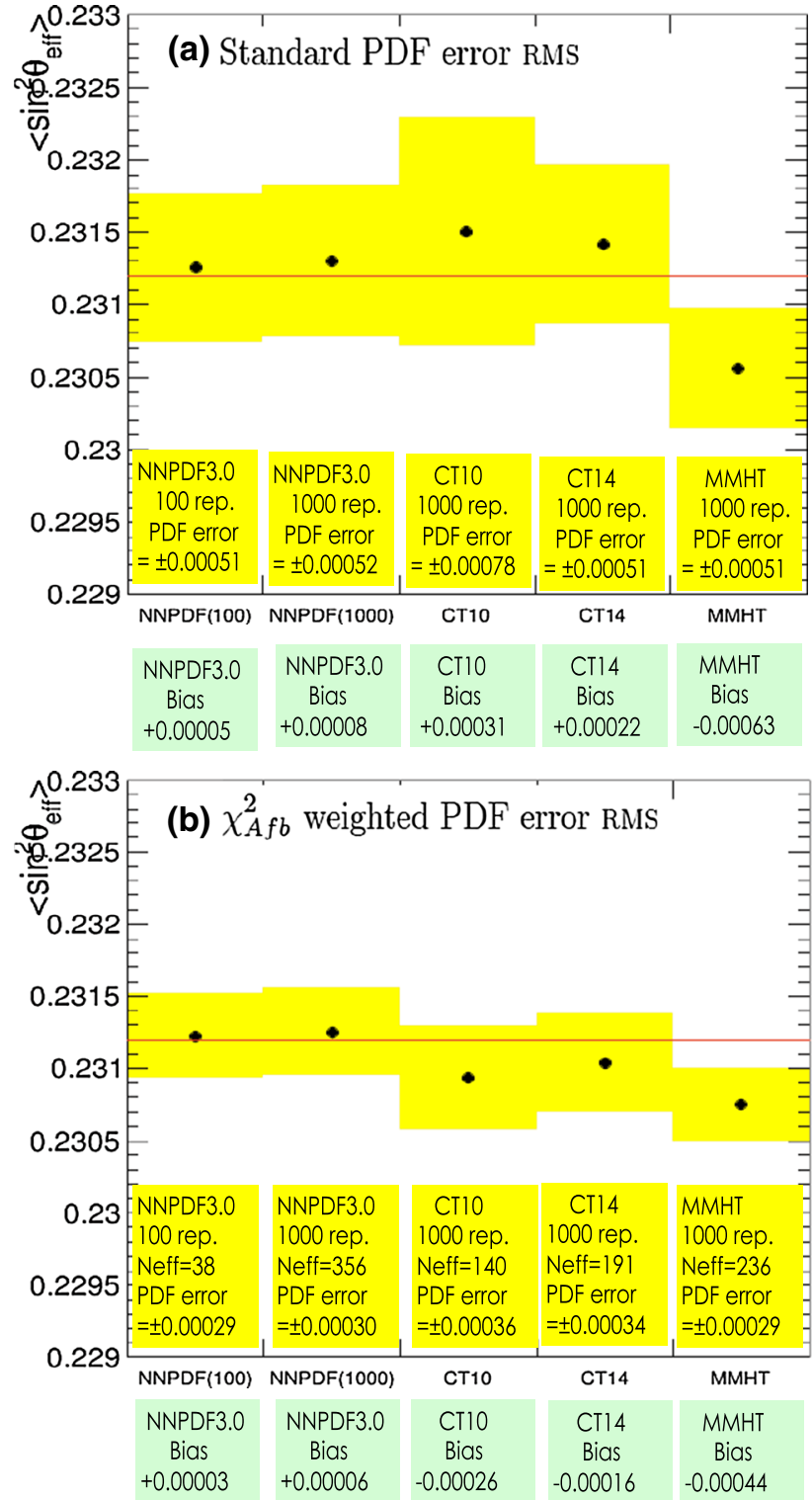

Fig. 10 The average of the results from the analyses of the 64 LHC pseudo experiments. Each pseudo experiment is analyzed with 100 NNPDF3.0 templates, 1000 NNPDF3.0 templates, 1000 CT10 templates and 1000 MHHT templates. The pseudo data for each experiment are generated by the POWHEG MC with the default NNPDF 3.0 (NLO) PDF and $\sin ^{2} \theta_{\text {eff }}=0.23120$. a Analysis using the standard mean and RMS of the $\sin ^{2} \theta_{\text {eff }}$ values extracted with each PDF set. b Analysis using the $\chi_{A f b}^{2}$ weighted mean and RMS of the $\sin ^{2} \theta_{\text {eff }}$ values extracted with each PDF set

1000 CT10 replicas and 1000 MHHT replicas. The standard mean and RMS (=PDF uncertainty) of the $\sin ^{2} \theta_{\text {eff }}$ values extracted with each PDF set are shown in Fig. 10a. The $\chi_{A f b}^{2}$ weighted mean and RMS( $=$ PDF uncertainty) of the $\sin ^{2} \theta_{\text {eff }}$ values extracted with each PDF set are shown in Fig. 10b.

As expected, since the pseudo data are generated with POWHEG with the default NNPDF 3.0 (NLO) PDF, the input value of $\sin ^{2} \theta_{\text {eff }}=0.23120$ is extracted with no bias when the pseudo data are analyzed using templates generated with
Table 4 Expected statistical and weighted PDFs uncertainties in the measurements of $\sin ^{2} \theta_{W}$ and $M_{W}^{\text {indirect }}$ with a CMS like detector for two samples. (a) A total of $15 \mathrm{M}$ reconstructed dilepton events $(8.2 \mathrm{M}$ $\mu^{+} \mu^{-}$and $6.8 \mathrm{M} e^{+} e^{-}$) in a CMS like detector. This is is similar to the existing $19 \mathrm{fb}^{-1} \mathrm{CMS}$ data sample at $8 \mathrm{TeV}$. (b) $120 \mathrm{M}$ reconstructed $\mu^{+} \mu^{-}$events, which the sample expected for a CMS like detector with $200 \mathrm{fb}^{-1}$ at $13-14 \mathrm{TeV}$

\begin{tabular}{lll}
\hline CMS like detector & 2016 sample & 2017-18 sample \\
\hline Energy & $8 \mathrm{TeV}$ & $13-14 \mathrm{TeV}$ \\
\hline Number of & $8.2 \mathrm{M} \mu^{+} \mu^{-}$ & $120 \mathrm{M} \mu^{+} \mu^{-}$ \\
Reconstructed events & $6.8 \mathrm{M} e^{+} e^{-}$ & - \\
\hline$\Delta \sin ^{2} \theta_{W}$ & & \\
Statistical error & \pm 0.00034 & \pm 0.00011 \\
Weighted PDF error & \pm 0.00022 & \pm 0.00014 \\
(Stat + PDF) error & \pm 0.00040 & \pm 0.00018 \\
$\Delta M_{W}^{\text {indirect }}$ & $\mathrm{MeV}$ & $\mathrm{MeV}$ \\
Statistical error & \pm 17 & \pm 5 \\
Weighted PDF error & \pm 11 & \pm 7 \\
(Stat + PDF) error & \pm 20 & \pm 9 \\
\hline
\end{tabular}

either 100 or 1000 NNPDF 3.0 (NLO) replicas. The PDF uncertainty is reduced from \pm 0.00052 to \pm 0.00030 when $\chi_{A f b}^{2}$ weighted mean and RMS are used.

The CT10 PDFs are less precise because they do not incorporate any LHC data. Consequently, the uncertainties with CT10 PDFs are larger. The CT10 PDF uncertainty is reduced from \pm 0.00078 to \pm 0.00036 when $\chi_{A f b}^{2}$ weighted mean and RMS are used. Similarly, the bias with CT10 is reduced from +0.00031 to -0.00026 which is within the reduced PDF uncertainty. The CT14 PDFs and MMHT PDFs incorporate LHC data in the fits. The PDF uncertainties with CT14 are reduced from \pm 0.00051 to \pm 0.00034 when $\chi_{A f b}^{2}$ weighted mean and RMS are used. Similarly, the bias with CT14 is reduced from +0.00022 to -0.00016 , which is within the reduced PDF uncertainty. The PDF uncertainties with MMHT are reduced from \pm 0.00051 to \pm 0.00029 with $\chi_{A f b}^{2}$ weighted mean and RMS. Here, the bias with MMHT is reduced from -0.00063 to -0.00044 , but it is still larger than the PDF uncertainty.

As shown in Fig. 9, the $A_{f b}$ analysis of the pseudo data illustrates that MMHT PDF set is not fully consistent with the NNPDF or with CT14 PDF set. A similar study with actual $A_{f b}$ data at $8 \mathrm{TeV}$ would be a first step in the investigation of the origin of the differences between the various PDF sets.

\section{Conclusion}

We show that measurements of the Drell-Yan forwardbackward charge asymmetry $\left(A_{F B}(M, y)\right)$ at hadron collid- 
ers provide a new powerful tool to reduce the PDF uncertainties in the measurement of electroweak parameters.

Table 4 summarizes the analysis for two samples. The first (labeled 2016) is a sample of $8.2 \mathrm{M} \mu^{+} \mu^{-}$and 6.8M $e^{+} e^{-}$ reconstructed events (with $M_{l l}>50 \mathrm{GeV}$ ) corresponding to an integrated luminosity of $19 \mathrm{fb}^{-1}$ for a CMS like detector at $8 \mathrm{TeV}$. This sample is similar to the existing $19 \mathrm{fb}^{-1} \mathrm{CMS}$ data sample at $8 \mathrm{TeV}$. The statistical error in the measurement of $\sin ^{2} \theta_{\text {eff }}$ for this sample is expected to be \pm 0.00034 , and the weighted PDF uncertainty is expected to be \pm 0.00022 . These are equivalent to a statistical error of $\pm 17 \mathrm{MeV}$ and a weighted PDF uncertainty of $\pm 11 \mathrm{MeV}$ in the indirect measurement of $M_{W}$.

With the larger number of $\mu^{+} \mu^{-}$events expected to be collected at 13-14 TeV, both the statistical errors and the weighted PDF uncertainties are expected to be smaller. About $120 \mathrm{M}$ reconstructed $\mu^{+} \mu^{-}$events (with $M_{\mu \mu}>50 \mathrm{GeV}$ ) are expected in a CMS like detector for an integrated luminosity of $200 \mathrm{fb}^{-1}$ at 13-14 TeV. For this sample (labeled 2017-18), as shown in the second column of Table 4, the expected statistical error in the indirect measurement of $M_{W}$ is $5 \mathrm{MeV}$, and the weighted PDF uncertainty is $\pm 7 \mathrm{MeV}$. These expected errors are smaller than the uncertainties in the most recent direct measurements of $M_{W}$.

Acknowledgments This work was support by the US Department of Energy, office of High Energy Physics under Grant Number DE-SC0008475 .

Open Access This article is distributed under the terms of the Creative Commons Attribution 4.0 International License (http://creativecomm ons.org/licenses/by/4.0/), which permits unrestricted use, distribution, and reproduction in any medium, provided you give appropriate credit to the original author(s) and the source, provide a link to the Creative Commons license, and indicate if changes were made.

Funded by SCOAP ${ }^{3}$.

\section{References}

1. H.-L. Lai, M. Guzzi et al. (CT10), Phys. Rev. D 82, 074024 (2010)

2. S. Dulat et al. (CT14), arXiv:1506.07443

3. A.D. Martin et al. (MSTW08), Eur. Phys. J. C 63, 189-285 (2009). arXiv:0901.0002
4. L.A. Harland-Lang et. al. (MMHT14), Eur. Phys. J. C 75(5), 204 (2015). arXiv:1412.3989

5. R.D. Ball et al. (NNPDF 2.3), Nucl. Phys. B 867, 244 (2013). arXiv: 1207.1303

6. R.D. Ball et al. (NNPDF 3.0), JHEP 1504, 040 (2015). arXiv: 1410.8849

7. S. Carrazza et al., An unbiased Hessian representation for Monte Carlo PDFs. arXiv: 1505.06736

8. ZEUS and H1 Collaborations (Cooper-Sarkar, A.M. for the collaboration) PoS EPS-HEP2011, 320 (2011). arXiv:1112.2107

9. S. Alekhin, J. Bluemlein, S. Moch (ABM), Phys. Rev. D 89(5), 054028 (2014). arXiv: 1310.3059

10. K.A. Olive et al. (PDG), Chin. Phys. C 38, 090001 (2014). http:// pdg.lbl.gov

11. S. Heinemeyer, W. Hollik, G. Weiglein, L. Zeune, JHEP 04, 84 (2013). arXiv: 1211.5142

12. T. Altonen et al. (CDF collaboration), Phys, Rev. D 88(2013), 072002 (2013). arXiv: 1307.0770

13. T. Altonen et al. (CDF collaboration), Phys. Rev. D 89, 072005 (2014). arXiv:1402.2239

14. V.M. Abazov et al. (D0 collaboration), Phys. Rev. Lett. 115, 041801 (2015). arXiv: 1408.5016

15. ATLAS collaboration, Measurement of the forward-backward asymmetry of electron and muon pair-production in pp collisions at $\sqrt{s}=7 \mathrm{TeV}$ with the ATLAS detector. arXiv:1503.03709

16. A. Bodek et al., Eur. Phys. J. C 72, 2194 (2012). arXiv: 1208.3710

17. A. Bodek, Eur. Phys. J. C 67, 321 (2010). arXiv:0911.2850

18. F. Landry, R. Brock, P.M. Nadolsky, C.-P. Yuan, Phys. Rev. D67 073016 (2003). http://hep.pa.msu.edu/resum/

19. S. Alioli, P. Nason, C. Oleari, E. Re, JHEP 1101, 095 (2011). arXiv: 1009.5594

20. G. Watt, R.S. Thorne (MRST), JHEP 08, 052 (2012). arXiv: 1205.4024

21. https://mstwpdf.hepforge.org/random/

22. W.T. Giele, S. Keller, Phys. Rev. D 58, 094023 (1998). arXiv:hep-ph/9803393

23. N. Sato, J.F. Owens, H. Prosper, Phys. Rev. D 89, 114020 (2014). arXiv: 1310.1089

24. H. Paukkunen, P. Zurita, PDF reweighting in the Hessian matrix approach. arXiv:1402.6623

25. R.D. Ball, V. Bertone, F. Cerutti, L. Del Debbio, S. Forte, A. Guffanti, J.I. Latorre, J. Rojo, M. Ubiali (NNPDF), Nucl. Phys. B 849, 112 (2011). arXiv: 1012.0836 